\title{
Carbon Footprint Determination When Using Residual Agricultural Biomass for Energy Production
}

\author{
Erol Murad
}

EKKO OFFICE AG, Innovation Department, Bucharest 024102, Romania

\begin{abstract}
At present, the focus is on distributed energy generation with low or negative carbon emissions as well as high conversion yields. In Romania, the renewable energy resource that can be used and produced when and wherever necessary is residual agricultural biomass with a potential of 31 million tons, which can produce over $40 \%$ of the national energy demand. Residual agricultural biomass is produced with an average energy efficiency of $6 \mathrm{kWh} \cdot \mathrm{bm} / \mathrm{kWh}$ input. The CHAB (combined heat and biochar production) concept produces high yield thermal energy as well as BC (biochar) with an average carbon footprint of $140 \mathrm{~kg} / \mathrm{ton}$ biomass. If the energy produced is used to produce agricultural output, the negative carbon footprint increases by reducing the consumption of fossil fuels. It increases energy independence, the safety of agricultural production, the number of jobs, and regional economic development.
\end{abstract}

Key words: Waste biomass, energy, $\mathrm{BC}, \mathrm{CHAB}$, carbon footprint.

\section{Introduction}

Agriculture has been and remains the main source of raw materials for food and industrialization. The concept of sustainable development and evidence of climate change tends to tackle the issue of adapting efficient and sustainable agricultural production technologies to humanity in order to provide food for an ever-growing population fed by a declining global agricultural area.

Sustainable development of agriculture also involves increasing the energy independence of agricultural farms by reducing fossil fuel consumption, increasing and maintaining productive soil capacity, and reducing use of mineral fertilizers in favor of compost, which is linked to current ecological requirements and leads to the need to increase the level of use of residual biomass resulting from agricultural crops $[1,2]$.

At present, direct biomass burning, chopped, briquetted or pelletized is the majority procedure. As an alternative to current methods of thermal energy production from biomass, it is proposed the $\mathrm{CHAB}$

Corresponding author: Erol Murad, associate professor, research fields: thermo-chemical gasification of biomass, modeling and simulation of unconventional processes. E-mail: erolmurad@yahoo.com. (combined heat and biochar production) concept which also includes the $\mathrm{BC}$ (biochar) generation. $\mathrm{BC}$ is a sterile organic material obtained from biomass pyrolysis in an oxygen-free environment or with a controlled gasification, with a neutral or alkaline $\mathrm{pH}$. It has a carbon content of $75-90 \%$ and is characterized by high porosity and adsorption capacity [2, 4].

$\mathrm{BC}$ is used to improve the long-term fertility of agricultural soils, and secondarily as a filtering agent for air, gas and water. Built in soil, it is the most economical and ecological way of sequestering at least $25 \%$ of carbon, for extended periods between 100 and 1,000 years; it also has many other applications in the most diverse fields of human activity $[3,5,6]$.

In order to evaluate how the waste biomass can be efficiently exploited, an energy balance and carbon mass analysis will be carried out, from which the carbon footprint can be calculated to determine the useful energy produced, and to create a base analysis and optimization of variants and energy conversion regimes.

In nature, spontaneous vegetation uses solar energy, carbon dioxide $\left(\mathrm{CO}_{2}\right)$ and soil fertility to produce a vegetal mass containing carbon $\mathrm{Cb}$, which, through the natural carbon circuit, returns to the atmosphere. 
Vegetable crop production has as its main product a biomass destined for human and zootechnical consumption, which we call food biomass, as well as a by-product called waste biomass (Fig. 1). Solar energy, carbon dioxide $\left(\mathrm{CO}_{2}\right)$ in the atmosphere, soil fertility, as well as Econs energy consumed to carry out agricultural works contribute to the achievement of vegetal agricultural production. This produces an energy accumulation in agricultural products - Ebal in the main product and Ebr in the wast biomass [7].

The efficiency of agricultural production is determined by the ratio of energy at exit $E \mathrm{~b}$ and that of input $E$ cons. Therefore energy efficiency is calculated with Eq. (1):

$$
E F E N=\frac{E b}{E c o n s}=\frac{E b a l+E b r}{E c o n s}
$$

Table 1 presents the energies produced and consumed for the main agricultural crops as well as the total energy efficiency EFEN, the main EFENp and the secondary EFENs [7].

It is noted that most of the agricultural production has an EFEN $>1$ overall efficiency and many crops also have an EFENs secondary efficiency of more than 3 , which confirms the conclusion that the agricultural crop production is also producing renewable energy with low costs and with a reduced CFPf (carbon footprint) [7].

Biomass produced has an Etotal energy for which Econs was consumed from fossil fuels. It is assumed that the fuel used is diesel fuel having a CFPf $=0.0815$ $\mathrm{kg} / \mathrm{kWh}$ footprint for base energy. The overall diesel fuel efficiency is estimated at $\eta_{\mathrm{f}}=0.864$, which results in a positive footprint in the CFPatm atmosphere $(\mathrm{kg} \cdot \mathrm{C} / \mathrm{kWh})$ for residual biomass:

$$
\text { CFPatm }=\frac{1}{E F E N} \frac{C F P f}{\eta_{f}}=0.0944
$$

Since for the production of Econs fossil fuels are consumed in the atmosphere, a quantity of $\mathrm{CfO}=$ Econs.CFPf is released.

Table 1 presents the energy efficiency values for the main crops in Romania. It is noticed that the majority of agricultural crops produce residual biomass with an EFENs $>>0$, so they are economically and

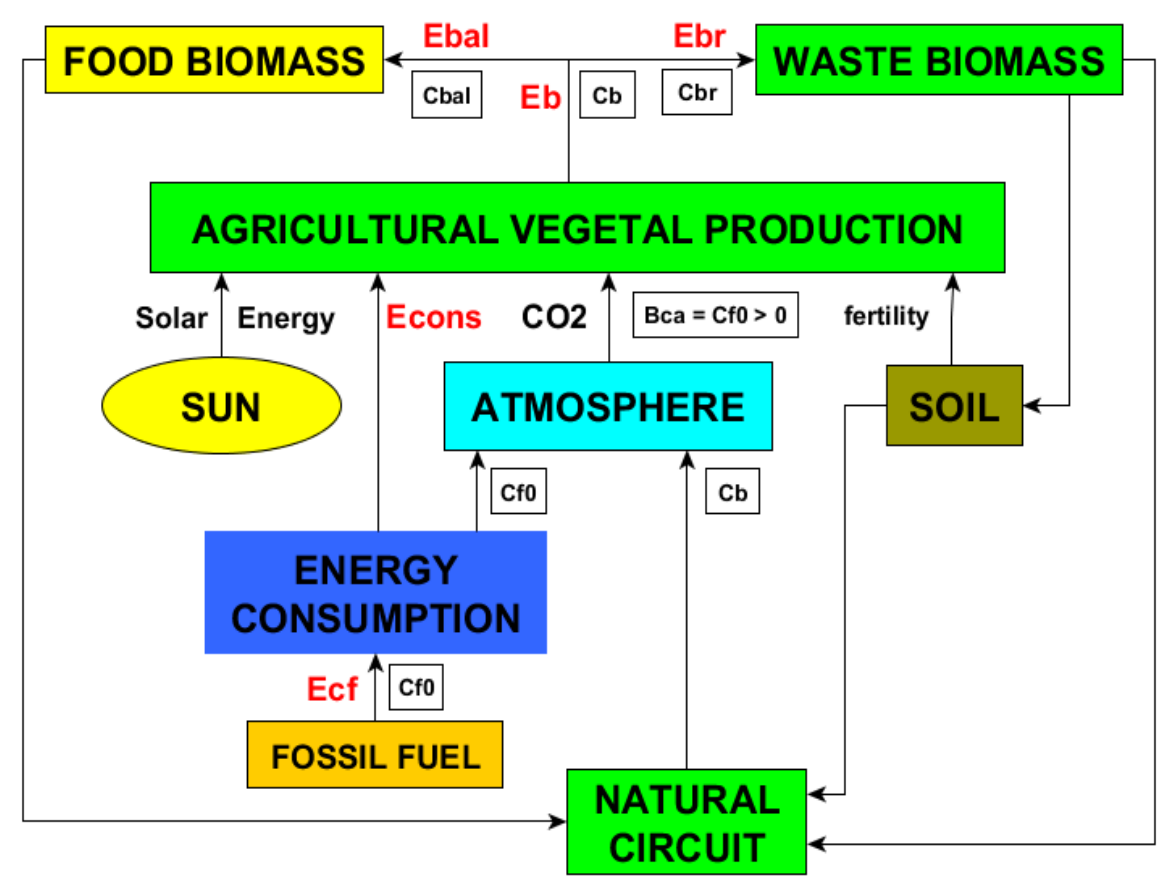

Fig. 1 Agricultural crop production general model. 
Table 1 Energies produced, consumed and energy efficiency for the main agricultural crops.

\begin{tabular}{llllllll}
\hline \multirow{2}{*}{ Crops } & \multicolumn{3}{c}{ Energy produced } & \multicolumn{2}{l}{ Energy consumed } & \multicolumn{2}{c}{ Energetic efficiency } \\
\cline { 2 - 8 } & Etotal & Ebal & Ebr & Econs & EFEN & \multirow{2}{*}{ EFENp } & EFENs \\
\cline { 2 - 7 } & $\mathrm{kWh} / \mathrm{ha}$ & $\mathrm{kWh} / \mathrm{ha}$ & $\mathrm{kWh} / \mathrm{ha}$ & $\mathrm{kWh} / \mathrm{ha}$ & 17.63 & 7.95 & 9.68 \\
Corn & 91,029 & 41,054 & 49,975 & 5,163 & 7.12 & 2.91 & 4.21 \\
Winter wheat & 41,017 & 16,773 & 24,244 & 5,764 & 6.52 & 3.25 & 3.27 \\
Beans & 21,227 & 10,585 & 10,642 & 3,254 & 3.98 & 1.00 & 2.98 \\
Sunflowers & 19,807 & 4,970 & 14,837 & 4,982 & 6.36 & 3.99 & 2.36 \\
Soy & 29,517 & 18,550 & 10,967 & 4,643 & 1.87 & 1.00 & 0.87 \\
Plum & 23,981 & 12,775 & 11,206 & 12,833 & 1.09 & 0.52 & 0.57 \\
Vineyard & 17,547 & 8,381 & 9,167 & 16,028 & 1.29 & 0.83 & 0.45 \\
Apple & 22,372 & 14,467 & 7,906 & 17,383 & &
\end{tabular}

Table 2 Carbon footprints and the utilization yields for the main fuels entering the energy production processes of the waste biomass.

\begin{tabular}{|c|c|c|c|c|}
\hline Feature & Symbol & UM & Value & Notes \\
\hline Diesel fuel foot print & CFPdf & $\mathrm{kg} \cdot \mathrm{C} / \mathrm{kWh}$ & 0.0815 & \\
\hline Corn stalk foot print & CFPbr & $\mathrm{kg} \cdot \mathrm{C} / \mathrm{kWh}$ & 0.0873 & Corn stalk \\
\hline Corn stalk pellets foot print & CFPcp & $\mathrm{kg} \cdot \mathrm{C} / \mathrm{kWh}$ & 0.0873 & Corn stalk pellets \\
\hline Carbon foot print for peleting & CFPcpr & $\mathrm{kg} \cdot \mathrm{C} / \mathrm{kWh}$ & 0.0082 & \\
\hline Syngas foot print [CFPsg] & & $\mathrm{kg} \cdot \mathrm{C} / \mathrm{kWh}$ & 0.1059 & From gasifier \\
\hline Conversion yeld for diesel burning & Kconv $\cdot f$ & adim & 0.864 & \\
\hline Conversion yeld for BM burning & Kconv $\cdot b m$ & adim & 0.786 & \\
\hline Conversion yeld for syngas burning & Kconv $\cdot g$ & $\operatorname{adim}$ & 0.746 & \\
\hline Global BM footprint & $\mathrm{CFPbmu}$ & $\mathrm{kg} \cdot \mathrm{C} / \mathrm{kWhu}$ & 0.112 & \\
\hline Global diesel footprint & CFPfu & $\mathrm{kg} \cdot \mathrm{C} / \mathrm{kWhu}$ & 0.095 & \\
\hline Global syngas footprint & CFPgu & $\mathrm{kg} \cdot \mathrm{C} / \mathrm{kWhu}$ & 0.142 & \\
\hline
\end{tabular}

ecologically productive renewable energy resources, mainly the corn and wheat crops that offer biomass for pelleting. These aspects regarding the fruit cuttings and of the vines were presented in the works [2, 8]. Further analysis will be developed for corn crop, which has the highest energy efficiency, for pellets produced from corn stalks.

For the energy and carbon balance, Table 2 shows the carbon footprints and the utilization yields for the main fuels entering the energy production processes of the waste biomass: diesel fuel, corn stalk biomass, corn stalk pellets, biochar and syngas [3, 4, 9, 11].

From the calculation of the carbon footprint produced using the primary energy of the residual corn mass, taking into account the part of the carbon footprint due to the energy input at the system entry, it results that for $1 \mathrm{MWh}$ of the residual corn stalk consumed $56.7 \mathrm{kWh}$ at input with a carbon footprint of $\max .4 .623 \mathrm{~kg} \cdot \mathrm{C} / \mathrm{MWh}$.

\section{Energy Conversion System with Burning Process}

To perform the analysis, a model (Fig. 2) of a corn stalk pellet energy production system was designed using burning processes. The conversion of the energy biomass into useful energy is achieved with a biomass energy system block, which enters an Ecs.inp energy consisting of Ebre as a Kbe part of the pellet biomass that has been pelletized and a carbon content Cbre. To perform the conversion process, an Ecs.act power is also introduced with a CFPcons footprint.

The output energy Ecs.out consists of the energy emitted in Ecs.ev environment and a useful energy that can be divided: one Ecs.c part can be consumed directly for the production of the vegetal agricultural production and another Ecs.u that feeds the energy consumers external to the analyzed system. Part of the input energy, which represents the losses of $E$ cs.ev, is 


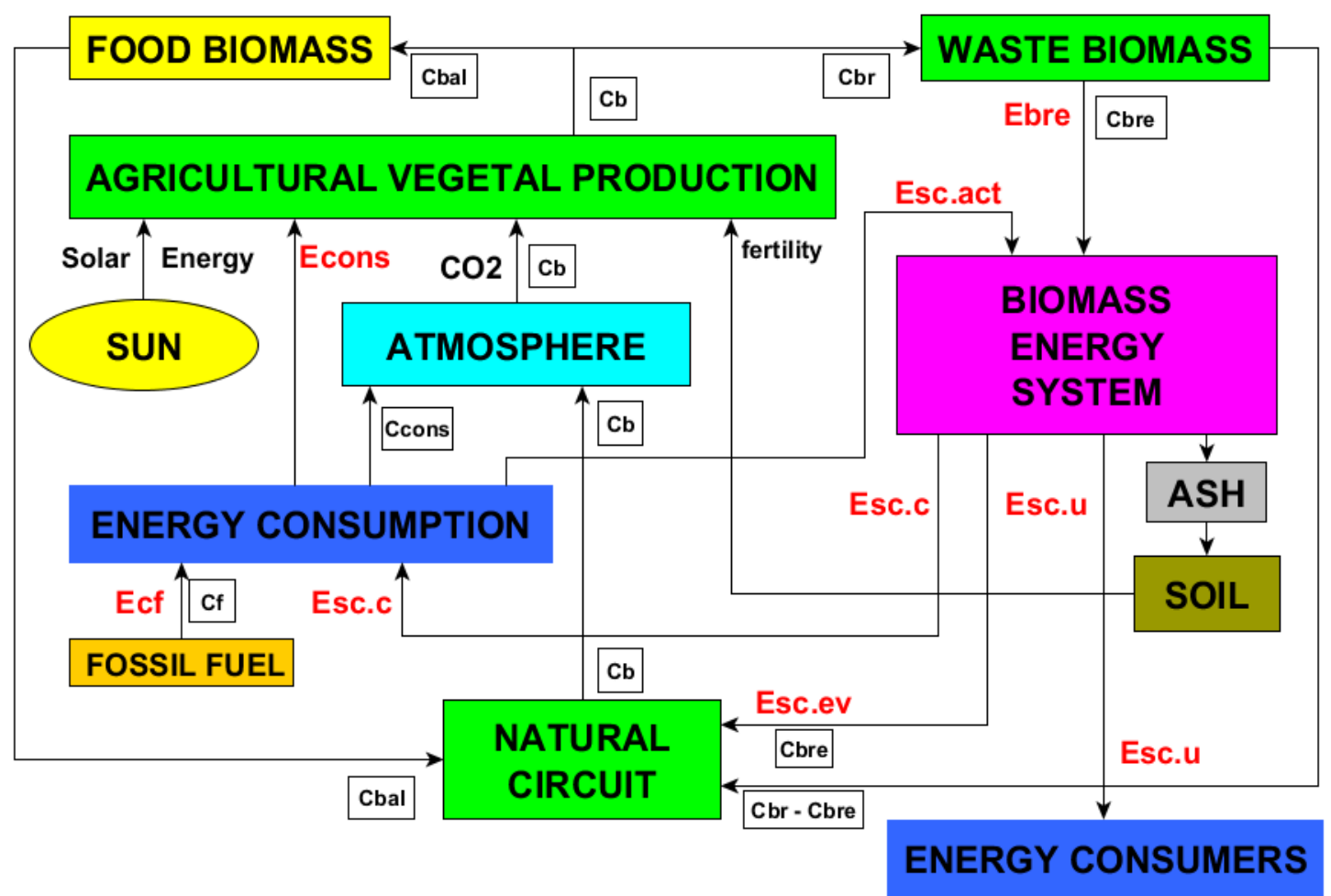

Fig. 2 Model of biomass energy system with burning process.

evacuated in the case of Cbre. Also comes the ASH without energy and carbon, which is incorporated into the soil.

The energy balance is:

$$
\Delta \text { Ebes }=\text { Ecs.inp }- \text { Ecs.out }=0
$$

Input energy is:

$$
\text { Ecs.inp }=\text { Ebre }+ \text { Ecs.act }
$$

Energy for system consumption is:

$$
\begin{gathered}
\text { Ecs.act }=\text { Ecs.pr }+ \text { Ecs.ard }+ \text { Ecs.he }= \\
\text { Kact } \cdot(\text { Kbe } \cdot \text { Ebre })
\end{gathered}
$$

where in the analyzed case, Kact $\approx 0.17$, resulting for Ecs.inp the relation:

$$
\begin{aligned}
\text { Ecs.inp }= & K b e \cdot E b r+K a c t(K b e \cdot E b r)= \\
& (1+K a c t)(K b e \cdot E b r)
\end{aligned}
$$

The output energies are: Ecs.ev—exhaust gas energy from the heat exchanger; Ecs.u-energy usable in external applications to the system; Ecs.c-energy consumed for the system by energy consumption. The energy relationship at the output is:

$$
\begin{gathered}
\text { Ecs.out }=\text { Ecs.ev }+(\text { Esc.u }+ \text { Ecs.c })= \\
\left(1-\eta_{c s}\right) \text { Ecs.out }+(E s c . u+E c s . c) \\
\text { where } \eta_{c s}=\eta_{p r} \cdot \eta_{\text {ard }} \cdot \eta_{h e}=
\end{gathered}
$$

$$
(1 / 1.1) \cdot 0.96 \cdot 0.9=0.7855 \cong 0.78
$$

The energy Ecs.c consumed in the system is determined by the take-off $\mathrm{Kbc}$ in the useful energy at the exit:

$E c s . c=K b c(E c s . o u t-E c s . e v)=K b c \cdot \eta_{s c} \cdot E c s . i n p=$ $K b c \cdot \eta_{s c} \cdot(1+K a c t) E b r e=K b c \cdot K c o n v \cdot(K b e \cdot E b r)$

$$
\text { where } \quad \text { Kconv }=\eta_{s c} \cdot(1+\text { Kact })
$$

The carbon balance shows that since the carbon footprint for $E$ cs.c $+E$ cs.u is incorporated into the exhaust outlet, it follows that: 


$$
\Delta \text { Cbes }=\text { Cbre }- \text { Csc.ev }=0
$$

It is noticed that Cbre re-enters the atmosphere through the combustion gases exhausted at the exchanger outlet. It follows that the carbon footprint for $E$ cs.u and $E$ cs.c is zero.

Another important block is the energy consumption subsystem. In block is the Ecf energy produced from fossil fuels with $\mathrm{Cf}$ carbon content and Ecs.c energy from the energy produced by the system with a zero footprint. The exit is $E$ cons = cnt. used directly for agricultural crop production and Ecs.act for the energy conversion system. The energy balance is:

$$
\Delta E e c=(E c f+E c s . c)-(E c o n s+E c s . a c t)=0
$$

Ecf energy from fossil fuels produces a positive carbon footprint.

$$
\begin{array}{r}
E c f=(\text { Econs }+ \text { Ecs.act })-\text { Ecs.c } \\
\text { where Econs }=\frac{\text { Etotal }}{E F E N} \cong \frac{E b r}{E F E N s}
\end{array}
$$

The carbon balance is:

$$
\begin{gathered}
\Delta C e c=C f-C \text { cons }=E c f \cdot C F P f-C \text { cons }=0 \\
\text { and } \text { Ccons }=C f
\end{gathered}
$$

For atmosphere carbon balance is:

$$
\Delta \text { Catm }=(C \text { cons }+C b)-C b=C \text { cons }
$$

Carbon footprint in atmosphere is:

$$
\begin{gathered}
C F P a t m=\frac{C c o n s}{E c o n s+E c s . c}= \\
\frac{C f}{E b r / E F E N s+E c s . c}=E c f \frac{C F P f}{E b r / E F E N s+E c s . c}
\end{gathered}
$$

If we want to get a zero fingerprint, CFPatm $=0$, then $E \mathrm{cf}=0$ and an Ecs.c energy is required in the system with the value:

$$
\begin{gathered}
\text { Ecs.c }=\text { Econs }+ \text { Ecs.act }= \\
E b r / E F E N s+\text { Kact } \cdot(\text { Kbe } \cdot \text { Ebr })= \\
E b r(1 / \text { EFENs }+ \text { Kact } \cdot \text { Kbe })
\end{gathered}
$$

If an Ecs.u useful power is required for applications, it is necessary to determine which Kbe quota of residual biomass to be harvested should be used.

$$
\text { Ecs.u }=(1-K b c) \cdot K c o n v \cdot(K b e \cdot E b r)
$$

$$
\text { from where } K b c=1-\frac{E s c . u}{K c o n v \cdot(K b e \cdot E b r)}
$$

Ecs.c $=\left(1-\frac{E s c . u}{K c o n v \cdot(K b e \cdot E b r)}\right) \cdot K c o n v \cdot(K b e \cdot E b r)=$

$$
K c o n v \cdot(K b e \cdot E b r)-E s c . u
$$

\begin{tabular}{|c|c|c|c|c|c|}
\hline Kbu & 0.100 & 0.200 & 0.300 & 0.400 & 0.512 \\
\hline$K$ be $\geq$ & 0.330 & 0.493 & 0.655 & 0.818 & 1.000 \\
\hline
\end{tabular}

And using Eq. (9) results:

$$
\begin{gathered}
\text { Ecs.c }=\text { Kconv } \cdot(\text { Kbe } \cdot \text { Ebr })-E s c . u= \\
E b r(1 / E F E N s+K a c t \cdot K b e)
\end{gathered}
$$

It is shared with $E$ br and it follows:

$$
\begin{gathered}
\text { Kconv } \cdot \text { Kbe }-E s c u / E b r= \\
1 / E F E N s+K a c t \cdot K b e
\end{gathered}
$$

In order to ensure the Ecs.u value required for the $K$ be harvested waste biomass, it must be greater than:

$$
\begin{gathered}
\text { Kbe } \geq \frac{1}{K c o n v-K a c t}\left(K b u+\frac{1}{E F E N S}\right) \\
\text { where } K b u=\frac{E s c . u}{E b r}
\end{gathered}
$$

Table 3 shows the values of the Kbe coefficient according to the $\mathrm{Kbu}$ share of the energy demand.

Table 3 Kbe coefficient according to the Kbu of the energy demand. 


\section{Energy Conversion System with CHAB Concept}

As previously described, the application of the CHAB concept leads to the production of energy and biochar. Fig. 3 presents a model for a system that produces bio-fuel and energy from pyrolysis or gasification processes from residual biomass $[2,4,9,10,12]$.

Tables 4 and 5 show the energies and carbon content of the input and output products.

The model shown in Fig. 3 has a biochar output with $C$ bch carbon content and contains an Ebch energy.

For energy from biomass $\mathrm{CHAB}$ system energy balance is:

$$
\Delta \text { Ebes }=\text { Ecs.inp }- \text { Ecs.out }=0
$$

Input energy is:

$$
\text { Ecs.inp }=\text { Ebre }+ \text { Ecs.act }
$$

$$
\begin{aligned}
E c s . i n p= & K b e \cdot E b r+K a c t(K b e \cdot E b r)= \\
& (1+K a c t)(K b e \cdot E b r)
\end{aligned}
$$

The relationship for the output energy is:

$$
\begin{gathered}
\text { Ecs.out }=\text { Ecs.ev }+(\text { Esc.u }+ \text { Ecs.c })+\text { Ebch }= \\
\left(1-\eta_{c s}\right)(\text { Ecs.out }- \text { Ebch })+ \\
(\text { Esc.u }+ \text { Ecs.c })+\text { Ebch }
\end{gathered}
$$

Because at the carbon balance the carbon footprint for $E$ cs.c $+E$ cs.u is included in the exhaust outlet it results that:

$$
\begin{gathered}
\Delta \text { Cbes }=\text { Cbre }-(\text { Csc.ev }+ \text { Cbch })= \\
\text { Cbre }-(\text { Csc.ev }+ \text { Kbch } \cdot \text { Cbre })=0 \\
\text { Csc.ev }=\text { Cbre }(1-\text { Kbch })
\end{gathered}
$$

In this case for atmosphere carbon balance is:

$$
\begin{gathered}
\Delta \text { Catm }=(\text { Ccons }+C b-C b c h)-C b= \\
\text { Ccons }- \text { Cbch }
\end{gathered}
$$

Table 4 Energies and carbon content of the input products.

\begin{tabular}{lllll}
\hline Feature & $\mathrm{UM}$ & Corn stalks pellets & Biochar & Pyrolysis gas \\
\hline Relative masse & real & 1.00 & 0.237 & 0.763 \\
Carbon & real & 0.4053 & 0.7267 & 30.55 \\
Oxygen & real & 0.3905 & 0.489 & 49.66 \\
Hydrogen & real & 0.0540 & 0.0126 & 6.69 \\
Ash & real & 0.0502 & 0.2118 & 0 \\
Humidity & real & 0.10 & 0.00 & 13.11 \\
L.H.V & $\mathrm{MJ} / \mathrm{kg}$ & 14.98 & 25.60 & 11.68 \\
Carbon content & $\%$ & 100 & 42.0 & 58.0 \\
Energy content & $\%$ & 100 & 40.50 & 59.50 \\
$\mathrm{CO}_{2}$ footprint & $\mathrm{kg} \cdot \mathrm{CO}_{2} / \mathrm{kWh}$ & 0.357 & 0.375 & 0.345 \\
Carbon footprint & $\mathrm{kg} \cdot \mathrm{C} / \mathrm{kWh}$ & 0.097 & 0.102 & 0.094 \\
\hline
\end{tabular}

Table 5 Energies and carbon content of the output products.

\begin{tabular}{lllll}
\hline Feature & $\mathrm{UM}$ & Corn stalks pellets & Biochar & Gasified biomass \\
\hline Relative masse & real & 1.00 & 0.157 & 0.843 \\
Carbon & real & 0.4053 & 0.6316 & 0.3632 \\
Oxygen & real & 0.3905 & 0.032 & 0.4573 \\
Hydrogen & real & 0.054 & 0.0167 & 0.0609 \\
Ash & real & 0.0502 & 0.3197 & 0 \\
Humidity & real & 0.100 & 0.00 & 0.1186 \\
L.H.V & $\mathrm{MJ} / \mathrm{kg}$ & 14.98 & 20.20 & 14.01 \\
Carbon content & $\%$ & 100 & 21.17 & 78.83 \\
Energy content & $\%$ & 100 & 24 & 76 \\
$\mathrm{CO}_{2}$ footprint & $\mathrm{kg} \cdot \mathrm{CO}_{2} / \mathrm{kWh}$ & 0.357 & 0.413 & 0.342 \\
Carbon footprint & $\mathrm{kg} \cdot \mathrm{C} / \mathrm{kWh}$ & 0.097 & 0.113 & 0.093 \\
\hline
\end{tabular}




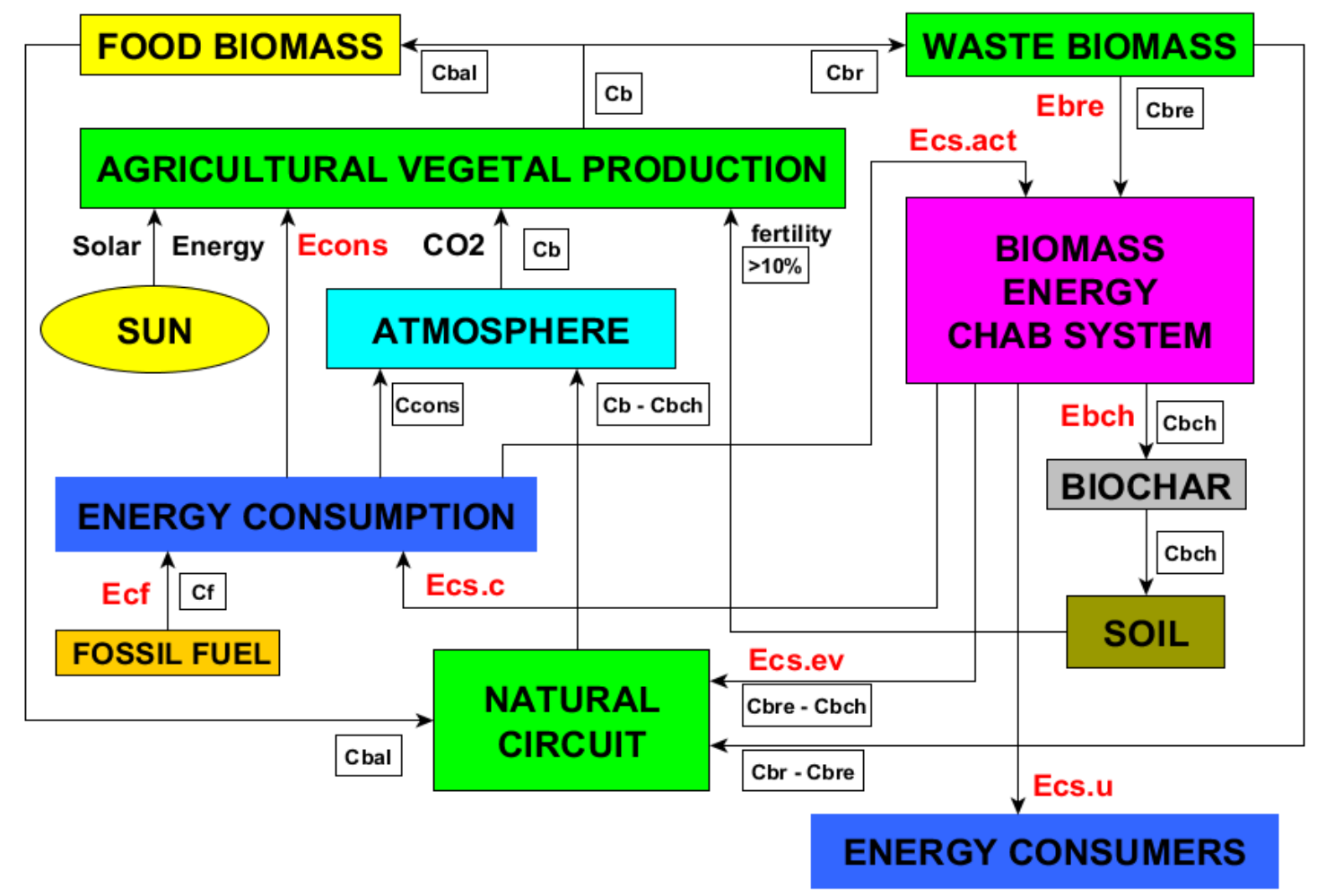

Fig. 3 Model of biomass energy system with CHAB concept.

Carbon footprint in atmosphere is:

$$
\begin{gathered}
C F P a t m=\frac{C c o n s-C b c h}{E c f+E c s . c}=\frac{C f-K b c h \cdot C b r e}{E c f+E c s . c}= \\
\frac{E c f \cdot C F P f-K b c h \cdot(K b e \cdot E b r) \cdot C F P b m}{E c f+E c s . c}
\end{gathered}
$$

Two situations are analyzed: CFPatm $=0$ or, for $E \mathrm{cf}$ $=0$, is obtained CFPatm $<0$.

For condition CFPatm $=0$ :

$E c f \cdot C F P f-K b c h \cdot(K b e \cdot E b r) \cdot C F P b m=0$

If $E$ cs.c $=0$ of the balance results $E$ cons $=E$ cf.

$$
\begin{gathered}
(\mathrm{Ebr} / \mathrm{EFENS}) \cdot \mathrm{CFPf}- \\
\mathrm{Kbe} \cdot(\mathrm{Kbch} \cdot \mathrm{CFPbm}) \cdot \mathrm{Ebr}=0
\end{gathered}
$$

To meet this condition, you must:

$$
\text { Kbe }=\frac{C F P f}{C F P b m} \cdot \frac{1}{K b c h \cdot E F E N S}
$$

For gasification results $K b_{\mathrm{g}} \geq 0.44$, and for pyrolysis $K \mathrm{be}_{\mathrm{p}} \geq 0.23$. When using gasification, more energy is available for external applications.

If the system energy $E$ cs.c $\approx E$ br/EFENs covers the energy requirement it results that $E \mathrm{cf}=0$ and the carbon footprint is negative:

$$
C F P a t m=-\frac{K b c h \cdot(K b e \cdot E b r) \cdot C F P b m}{(E b r / E F E N s)}=
$$

$$
\text { -Kbch.Kbe.CFPbm. EFENs }
$$

For $K$ be $=0.67$ with gasification $\mathrm{CFPatm}_{\mathrm{g}}=-0.124$, and with pyrolysis CFPatm $_{p}=-0.814$. Pyrolysis has to be noted the high negative value of the footprint, but there is a very little energy for the outside.

\section{Conclusions}

(1) Original models for energy conversion systems of agricultural waste biomass were developed by burning, pyrolysis and gasification processes, for the determination of energy and carbon balance, as well as 
of the carbon footprint in the atmosphere. A power conection of vegetable production with energy generated by the system has been introduced to reduce positive carbon footprint.

(2) The models were used to determine the regimes where the carbon footprint can be reduced to zero in the combustion process for different levels of useful energy needed for other applications.

(3) The simulation was performed for residual biomass from corn with the highest total energy efficiency $\mathrm{EFEN}=17.63$ and the energy-producing biomass for EFENs = 9.68. An energy use factor $K$ be $\in$ $(0,1)$ was used.

(4) In the system with burning process if $50 \%$ of the harvested residual biomass is used, for a zero footprint, is obtained useful energy for other thermal applications of about $80 \%$ of the biomass used.

(5) In system with gasification process for zero carbon footprint is necesary $K b \mathrm{e}_{\mathrm{g}} \geq 0.44$, and for a $K \mathrm{be}_{\mathrm{g}}=0.67$ is a negative footprint $\mathrm{CFPatm}_{\mathrm{g}}=-0.124$ $\mathrm{kg} \cdot \mathrm{C} / \mathrm{kWh}$, relatively small, less biochor is obtained but available more power for other applications.

(6) In system with pyrolysis process for zero carbon footprint is necesary $K b \mathrm{e}_{\mathrm{p}} \geq 0.44$, and for a $K \mathrm{be}_{\mathrm{p}}=0.67$ is a negative footprint $\mathrm{CFPatm}_{\mathrm{p}}=-0.814 \mathrm{~kg} \cdot \mathrm{C} / \mathrm{kWh}$, is a remarkable value due to the production of more biochar with a higher content of carbon.

(7) Developed models are a useful tool for the design of energy conversion systems for biomass in general and especially for agricultural waste biomass. It is a very useful tool in the development of automated control systems, both as structure and optimal control algorithms.

(8) Economic aspects will also be attached to become the most complete tool for developing biomass energy conversion systems.

\section{References}

[1] Christoph, S. 2010. "Biochar from Agricultural and
Forestry Residues - A Complementary Use of 'Waste' Biomass." In U.S.-Focused Biochar Report, Assessment of Biochar's Benefits for the United States of America, edited by J. Levine, USBI and CEES, Colorado, USA.

[2] Murad, E. 2013. "CHAB from Agricultural Biomass with TLUD, Scientific Communications Session, ICEDIMPH-HORTING.” Bucureşti, 28 November 2013.

[3] McLaughlin, H. 2011. "Combined Heat and Biochar: An Alternate Economic Option for Renewable Biomass." Presented at the 3rd Annual Conference of Heating the Northeast with Renewable Biomass, 15 April 2011.

[4] Murad, E., Dumitrescu, C., Dragomir, F., and Popescu, M. 2016. "CHAB Concept in Sustainable Development of Agriculture." In Proceedings of the International Symposium ISB-INMA 2016, ISSN 2344-4118, INMA Bucuresti, 257-62.

[5] Schmidt, H. P., et al. 2012. "European Biochar Certificate-Guidelines for Biochar Production." Presented at 2012 Delinat Institute und Biochar Science Network, Version 4.2 of 13 June 2012.

[6] Peters, J. F., Iribarren, D., and Dufour, J. 2015. "Biomass Pyrolysis for Biochar or Energy Application's a Life Cycle Assessment.” Envir. Sci. Technol. 49 (8): 5195-202.

[7] Răus, L., and Jităreanu, G. 2011. "The Energetic Efficiency of Different Tillage System and Fertilization Levels." Presented at Soil Minimum Tillage Systems, 6th International Symposium, Cluj-Napoca, 27-29 June 2011.

[8] Murad, E. 2014. "CHAB Concept in Viticulture." Presented at Scientific Communications Session ICDVV Valea Călugărescă, 12 June 2014 (in Romania).

[9] Murad, E., and Dragomir, F. 2012. "Heat Generators with TLUD Gasifier for Generating Energy from Biomass with a Negative Balance of $\mathrm{CO}_{2}$." In Proceedings of the 2012 International Conference HERVEX, 7-9 November 2012, Călimăneşti-Căciulata Romania.

[10] Murad, E. 2013. "Biomass Cogeneration Systems with Steam Cycle for Convective Dryers with Energy Independence and Negative Balance of $\mathrm{CO}_{2}$." Presented at CIEM 2013, UPBBucureşti, 7-8 November 2013.

[11] Whitfield, J. 2013. "Combined Heat and Biochar Production." Whitfield Biochar. USA, WA.

[12] Murad, E. 2016. "Power Production a Negative Carbon Balance with CHAB Concept." Presented at International Conference HERVEX 2016, Govora, 9-11 November 2016. 